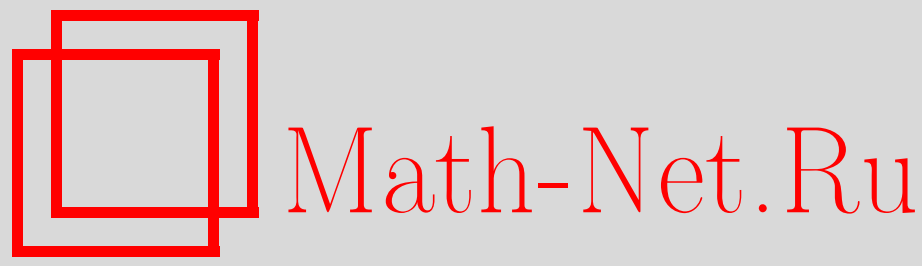

Н. А. Славнов, Детерминантные представления для скалярных произведений в алгебраическом анзаце Бете, TMФ, 2018, том 197, номер 3, 435-443

DOI: https://doi.org/10.4213/tmf9623

Использование Общероссийского математического портала Math-Net.Ru подразумевает, что вы прочитали и согласны с пользовательским соглашением http://www.mathnet.ru/rus/agreement

Параметры загрузки:

IP: 54.237 .59 .107

26 апреля 2023 г., 14:07:52

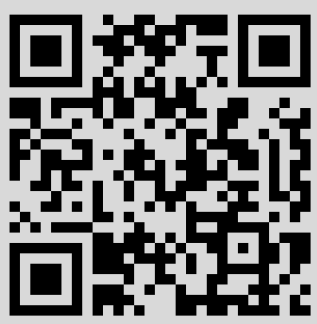




\title{
ДЕТЕРМИНАНТНЫЕ ПРЕДСТАВЛЕНИЯ ДЛЯ СКАЛЯРНЫХ ПРОИЗВЕДЕНИЙ В АЛГЕБРАИЧЕСКОМ АНЗАЦЕ БЕТЕ
}

\begin{abstract}
Рассматриваются модели с $\mathfrak{g l}(2 \mid 1)$-симметрией, решаемые с помощью вложенного алгебраического анзаца Бете. Получено новое детерминантное представление для скалярного произведения твистованного и обычного on-shell векторов Бете. Полученное представление приводит к новой формуле для скалярного произведения в моделях с $\mathfrak{g l}(2)$-симметрией.
\end{abstract}

Ключевые слова: алгебраический анзац Бете, скалярное произведение, градуированные модели.

DOI: https://doi.org/10.4213/tmf9623

\section{1. ВВЕДЕНИЕ}

Проблема вычисления скалярных произведений является крайне важной в алгебраическом анзаце Бете [1]-[3]. В рамках этого метода скалярное произведение представляет собой необходимый инструмент для вычисления формфакторов и корреляционных функций. Первые результаты в этом направлении для моделей с $\mathfrak{g l}(2)-$ инвариантной $R$-матрицей и ее $q$-деформацией были получены в [4]-[6]. Однако это явное представление (формула Изергина-Корепина) было весьма неудобно для приложений. Следующий шаг был сделан в статье [7], где было найдено детерминантное представление для частного случая скалярных произведений. В этом конкретном случае один из векторов является вектором общего вида, а другой - on-shell вектором Бете (т. е. собственным вектором трансфер-матрицы). Детерминантное представление для скалярных произведений открыло путь к изучению формфакторов и корреляционных функций в моделях с $\mathfrak{g l}(2)$-симметрией и их $q$-деформациями [8]-[13]. Оно также оказалось полезным для численного анализа корреляционных функций [14]-[17].

Исследование выполнено за счет гранта Российского научного фонда (проект № 14-50-00005).

* Математический институт им. В. А. Стеклова Российской академии наук, Москва, Россия. E-mail: nslavnov@mi-ras.ru 
Аналогичные результаты были недавно получены для моделей с $\mathfrak{g l}(3)-$ и $\mathfrak{g l}(2 \mid 1)$ симметриями [18]-[20]. В этих случаях, однако, приходится налагать более жесткие ограничения на векторы Бете. В частности, до сих пор не удалось вывести аналог представления из работы [7] для скалярного произведения on-shell вектора Бете и произвольного вектора. Тем не менее найденные детерминантные формулы для частных случаев скалярных произведений позволили получить компактные представления для формфакторов локальных операторов [21]-[26].

В настоящей работе мы выводим новое детерминантное представление для скалярного произведения твистованного и обычного on-shell векторов Бете в моделях с $\mathfrak{g l}(2 \mid 1)$-инвариантной $R$-матрицей ${ }^{1)}$. Новое представление является более удобным для численного анализа, чем представление работы [20], так как оно содержит детерминант матрицы меньшего порядка. В частном случае мы также получаем неизвестную ранее формулу для скалярного произведения векторов Бете в моделях c $\mathfrak{g l}(2)$-инвариантной $R$-матрицей. Данная формула может быть использована при вычислении термодинамического предела формфакторов и коэффициентов асимптотического разложения корреляционных функций.

Статья построена следующим образом. В разделе 2 мы описываем рассматриваемую модель, а также вводим соглашение об обозначениях. Раздел 3 содержит основные результаты статьи. Здесь мы приводим детерминантное представление для специального случая скалярного произведения векторов Бете и рассматриваем важный частный случай.

\section{2. ОПИСАНИЕ МОДЕЛИ}

2.1. Модели с $\mathfrak{g l}(2 \mid 1)$-симметрией. $R$-матрица $\mathfrak{g l}(2 \mid 1)$-инвариантных моделей действует в тензорном произведении $\mathbb{C}^{2 \mid 1} \otimes \mathbb{C}^{2 \mid 1}$, где $\mathbb{C}^{2 \mid 1}$ является $\mathbb{Z}_{2}$-градуированным векторным пространством с градуировкой $[1]=[2]=0,[3]=1$. Матрицы, действующие в этом пространстве, также градуированы: $\left[e_{i j}\right]=[i]+[j]$, где $e_{i j}$ суть элементарные единицы: $\left(e_{i j}\right)_{a b}=\delta_{i a} \delta_{j b} . R$-матрица имеет вид

$$
R(u, v)=\mathbb{I}+g(u, v) P, \quad g(u, v)=\frac{c}{u-v},
$$

где $\mathbb{I}$ - единичная матрица, $P$ - градуированный оператор перестановки [27], а $c$ некоторая константа.

Элементы матрицы мондромии $T(u)$ градуированы так же, как матрицы $\left[e_{i j}\right]$ : $\left[T_{i j}(u)\right]=[i]+[j]$. Их коммутационные соотношения задаются $R T T$-соотношением

$$
R(u, v)(T(u) \otimes \mathbb{I})(\mathbb{I} \otimes T(v))=(\mathbb{I} \otimes T(v))(T(u) \otimes \mathbb{I}) R(u, v),
$$

где тензорные произведения в пространствах $\mathbb{C}^{2 \mid 1}$ градуированы следующим образом:

$$
\left(\mathbb{I} \otimes e_{i j}\right) \cdot\left(e_{k l} \otimes \mathbb{I}\right)=(-1)^{([i]+[j])([k]+[l])} e_{k l} \otimes e_{i j}
$$

Равенство (2.2) имеет место в тензорном произведении $\mathbb{C}^{2 \mid 1} \otimes \mathbb{C}^{2 \mid 1} \otimes \mathcal{H}$, где $\mathcal{H}$ гильбертово пространство рассматриваемого гамильтониана.

\footnotetext{
1) См. п. 2.2 для определения твистованных on-shell векторов Бете.
} 
Градуированная трансфер-матрица определяется как суперслед матрицы монодромии:

$$
\mathcal{T}(u)=\operatorname{str} T(u)=\sum_{j=1}^{3}(-1)^{[j]} T_{j j}(u) .
$$

Благодаря соотношению $[\mathcal{T}(u), \mathcal{T}(v)]=0$ она задает некоторую интегрируемую систему.

2.2. Векторы Бете. Векторы Бете принадлежат пространству $\mathcal{H}$, в котором действует гамильтониан данной модели. В настоящей работе мы не используем явный вид этих векторов, однако читатель может найти его в работах [28], [29]. Дуальные векторы Бете принадлежат дуальному пространству $\mathcal{H}^{*}$.

Мы будем обозначать векторы Бете и дуальные к ним векторы соответственно через $\mathbb{B}_{a, b}(\bar{u} ; \bar{v})$ и $\mathbb{C}_{a, b}(\bar{u} ; \bar{v})$. Они параметризуются двумя наборами комплексных параметров (параметры Бете) $\bar{u}=\left\{u_{1}, \ldots, u_{a}\right\}$ и $\bar{v}=\left\{v_{1}, \ldots, v_{b}\right\}$, где $a, b=0,1, \ldots$. Если параметры Бете являются произвольными комплексными числами, то мы говорим, что соответствующий (дуальный) вектор Бете является вектором общего вида. Если оба набора $\bar{u}$ и $\bar{v}$ пусты, то $\mathbb{B}_{0,0}(\varnothing ; \varnothing)=|0\rangle$ и $\mathbb{C}_{0,0}(\varnothing ; \varnothing)=\langle 0|$, где $|0\rangle$ и $\langle 0|$ называются соответственно вакуумным и дуальным вакуумным векторами. Векторы $|0\rangle$ и $\langle 0|$ являются сингулярными векторами матричных элементов матрицы монодромии:

$$
\begin{aligned}
& T_{i i}(u)|0\rangle=\lambda_{i}(u)|0\rangle, \quad\langle 0| T_{i i}(u)=\lambda_{i}(u)\langle 0|, \quad 1 \leqslant i \leqslant 3, \\
& T_{j i}(u)|0\rangle=0, \quad\langle 0| T_{i j}(u)=0, \quad 1 \leqslant i<j \leqslant 3,
\end{aligned}
$$

где $\lambda_{i}(u)$ суть некоторые скалярные функции. В рамках обобщенной модели [4], рассматриваемой в настоящей статье, они остаются свободными функциональными параметрами. Ниже нам будет удобно иметь дело с отношениями этих функций

$$
r_{1}(u)=\frac{\lambda_{1}(u)}{\lambda_{2}(u)}, \quad r_{3}(u)=\frac{\lambda_{3}(u)}{\lambda_{2}(u)} .
$$

(Дуальный) вектор Бете становится собственным вектором трансфер-матрицы $\mathcal{T}(w)$, если параметры Бете удовлетворяют системе уравнений Бете

$$
\begin{array}{ll}
r_{1}\left(u_{j}\right)=\prod_{\substack{k=1, k \neq j}}^{a} \frac{f\left(u_{j}, u_{k}\right)}{f\left(u_{k}, u_{j}\right)} \prod_{l=1}^{b} f\left(v_{l}, u_{j}\right), & j=1, \ldots, a, \\
r_{3}\left(v_{j}\right)=\prod_{l=1}^{a} f\left(v_{j}, u_{l}\right), & j=1, \ldots, b,
\end{array}
$$

где мы ввели функцию

$$
f(u, v)=1+g(u, v)=\frac{u-v+c}{u-v} .
$$

Мы называем такие векторы on-shell векторами Бете (или дуальными on-shell векторами Бете). 
Помимо обычной матрицы монодромии удобно также рассматривать твистованную матрицу монодромии $T_{\kappa}(u)$ [5], [9], [18], [22]. Для моделей с $\mathfrak{g l}(2 \mid 1)$-симметрией она определяется следующим образом. Пусть $\kappa=\operatorname{diag}\left(\kappa_{1}, \kappa_{2}, \kappa_{3}\right)$ - диагональная матрица размера $3 \times 3$, где $\kappa_{i}$ суть комплексные числа. Тогда $T_{\kappa}(u)=\kappa T(u)$, где $T(u)$ - стандартная матрица монодромии.

Легко проверить, что твистованная матрица монодромии удовлетворяет $R T T$ соотношению (2.2) с $R$-матрицей (2.1). Суперслед твистованной матрицы монодромии $\mathcal{T}_{\kappa}(u)=\operatorname{str} T_{\kappa}(u)$ называется твистованной трансфер-матрицей. Собственные состояния (дуальные собственные состояния) твистованной трансфер-матрицы называются твистованными on-shell векторами Бете (соответственно твистованными дуальными on-shell векторами Бете). Твистованный (дуальный) вектор Бете общего вида становится твистованным (дуальным) on-shell вектором Бете, если параметры Бете удовлетворяют системе твистованных уравнений Бете

$$
\begin{array}{ll}
r_{1}\left(u_{j}\right)=\frac{\kappa_{2}}{\kappa_{1}} \prod_{\substack{k=1, k \neq j}}^{a} \frac{f\left(u_{j}, u_{k}\right)}{f\left(u_{k}, u_{j}\right)} \prod_{l=1}^{b} f\left(v_{l}, u_{j}\right), & j=1, \ldots, a, \\
r_{3}\left(v_{j}\right)=\frac{\kappa_{2}}{\kappa_{3}} \prod_{l=1}^{a} f\left(v_{j}, u_{l}\right), & j=1, \ldots, b .
\end{array}
$$

2.3. Обозначения. В настоящей работе мы пользуемся теми же обозначениями и соглашениями, что и в работе [20]. Напомним их.

Помимо функций $g(u, v)$ и $f(u, v)$, описанных выше, мы введем две функции

$$
\begin{aligned}
& h(u, v)=\frac{f(u, v)}{g(u, v)}=\frac{u-v+c}{c} \\
& t(u, v)=\frac{g(u, v)}{h(u, v)}=g(u, v)-\frac{1}{h(u, v)}=\frac{c^{2}}{(u-v)(u-v+c)} .
\end{aligned}
$$

Сформулируем теперь соглашение об обозначениях. Мы обозначаем наборы переменных чертой: $\bar{x}, \bar{u}, \bar{v}$ и т. д. Отдельные элементы наборов обозначаются латинским нижним индексом: $v_{j}, u_{k}$ и т. д. Как правило, количество элементов в наборах явно не указано, однако мы задаем его в специальных комментариях к формулам.

Для того чтобы сделать формулы более компактными, мы используем сокращенные обозначения для произведений функций, зависящих от двух переменных. А именно, если функции $g, f, h$ зависят от набора переменных, это означает, что следует взять произведение по соответствующему набору. Например,

$$
f\left(u_{k}, \bar{v}\right)=\prod_{v_{l} \in \bar{v}} f\left(u_{k}, v_{l}\right), \quad g\left(\bar{v}^{C}, \bar{v}^{B}\right)=\prod_{v_{j}^{C} \in \bar{v}^{C}} \prod_{v_{k}^{B} \in \bar{v}^{B}} g\left(v_{j}^{C}, v_{k}^{B}\right) .
$$

По определению любое произведение по пустому множеству равно 1. Двойное произведение равно 1, если хотя бы один из наборов пуст. Мы также вводим специальные обозначения для произведений функций $g$ :

$$
\Delta_{n}^{\prime}(\bar{u})=\prod_{j<k}^{n} g\left(u_{j}, u_{k}\right), \quad \Delta_{n}(\bar{v})=\prod_{j>k}^{n} g\left(v_{j}, v_{k}\right) .
$$

Легко видеть, что $\Delta_{n}^{\prime}(\bar{u})=(-1)^{n(n-1) / 2} \Delta_{n}(\bar{u})$. 


\section{3. ДЕТЕРМИНАНТНОЕ ПРЕДСТАВЛЕНИЕ ДЛЯ СКАЛЯРНОГО ПРОИЗВЕДЕНИЯ}

Скалярное произведение векторов Бете определяется как

$$
S_{a, b} \equiv S_{a, b}\left(\bar{u}^{C} ; \bar{v}^{C} \mid \bar{u}^{B} ; \bar{v}^{B}\right)=\mathbb{C}_{a, b}\left(\bar{u}^{C} ; \bar{v}^{C}\right) \mathbb{B}_{a, b}\left(\bar{u}^{B} ; \bar{v}^{B}\right)
$$

где все параметры Бете априори являются комплексными числами в общем положении. Мы добавили верхние индексы $C$ и $B$ к наборам $\bar{u}, \bar{v}$, дабы подчеркнуть, что векторы $\mathbb{C}_{a, b}$ и $\mathbb{B}_{a, b}$ могут зависеть от различных наборов параметров.

Рассмотрим скалярное произведение (3.1), где параметры Бете $\left\{\bar{u}^{B}, \bar{v}^{B}\right\}$ удовлетворяют системе (2.7), а параметры $\left\{\bar{u}^{C}, \bar{v}^{C}\right\}$ удовлетворяют системе (2.9). Тогда $\mathbb{B}_{a, b}\left(\bar{u}^{B} ; \bar{v}^{B}\right)$ является on-shell вектором Бете, а $\mathbb{C}_{a, b}\left(\bar{u}^{C} ; \bar{v}^{C}\right)$ - дуальный твистованный on-shell вектор Бете.

ПРЕДЛОЖЕНИЕ 3.1. Пусть $z_{i}-$ корни уравнения

$$
1=\frac{\kappa_{2}}{\kappa_{3}} \frac{f\left(z_{i}, \bar{u}^{C}\right)}{f\left(z_{i}, \bar{u}^{B}\right)}
$$

$u$

$$
\alpha_{i}=\frac{1}{\sum_{n=1}^{a}\left(t\left(z_{i}, u_{n}^{B}\right)-t\left(z_{i}, u_{n}^{C}\right)\right)} \frac{g\left(z_{i}, \bar{u}^{B}\right)}{g\left(z_{i}, \bar{u}^{C}\right)} \frac{g\left(z_{i}, \bar{v}^{C}\right)}{g\left(z_{i}, \bar{v}^{B}\right)} .
$$

Тогда скалярное произведение $S_{a, b}$ (3.1) имеет следующее детерминантное представление:

$$
S_{a, b}=H \operatorname{det}_{a}\left(\sum_{i=1}^{2 a} \alpha_{i} t\left(z_{i}, u_{j}^{C}\right)\left(g\left(u_{k}^{B}, z_{i}\right)+\frac{\kappa_{1}}{\kappa_{3} h\left(z_{i}, u_{k}^{B}\right)}\right)\right)
$$

где

$$
H=\left(\frac{\kappa_{3}}{\kappa_{1}}\right)^{a} h\left(\bar{u}^{B}, \bar{u}^{B}\right) \frac{g\left(\bar{v}^{B}, \bar{u}^{B}\right)}{g\left(\bar{v}^{C}, \bar{u}^{B}\right)} \Delta_{a}^{\prime}\left(\bar{u}^{C}\right) \Delta_{a}\left(\bar{u}^{B}\right) g\left(\bar{v}^{C}, \bar{v}^{B}\right) \prod_{k=1}^{b}\left(f\left(v_{k}^{C}, \bar{u}^{B}\right)-\frac{\kappa_{2}}{\kappa_{3}} f\left(v_{k}^{C}, \bar{u}^{C}\right)\right) .
$$

В частном случае $\bar{v}^{C}=\bar{v}^{B}=\varnothing, \kappa_{2}=\kappa$ и $\kappa_{1}=\kappa_{3}=1$ мы имеем дело с $\mathfrak{g l}(2)$ симметричными моделями. Тогда представление (3.4) упрощается.

ПРЕДЛОЖЕНИЕ 3.2. Пусть $z_{i}-$ корни уравнения

$$
1=\kappa \frac{f\left(z_{i}, \bar{u}^{C}\right)}{f\left(z_{i}, \bar{u}^{B}\right)}
$$

$u$

$$
\alpha_{i}=\frac{1}{\sum_{n=1}^{a}\left(t\left(z_{i}, u_{n}^{B}\right)-t\left(z_{i}, u_{n}^{C}\right)\right)} \frac{g\left(z_{i}, \bar{u}^{B}\right)}{g\left(z_{i}, \bar{u}^{C}\right)} .
$$

В этом случае скалярное произведение твистованного дуального оп-shell вектора Бете $\mathbb{C}_{a}\left(\bar{u}^{C}\right)=\mathbb{C}_{a, 0}\left(\bar{u}^{C} ; \varnothing\right)$ u on-shell вектора Бете $\mathbb{B}_{a}\left(\bar{u}^{B}\right)=\mathbb{B}_{a, 0}\left(\bar{u}^{B} ; \varnothing\right)$ uмеет следующее детерминантное представление:

$$
\mathbb{C}_{a}\left(\bar{u}^{C}\right) \mathbb{B}_{a}\left(\bar{u}^{B}\right)=\Delta_{a}^{\prime}\left(\bar{u}^{C}\right) \Delta_{a}\left(\bar{u}^{B}\right) h\left(\bar{u}^{B}, \bar{u}^{B}\right) \operatorname{det}_{a}\left(\sum_{i=1}^{2 a} \alpha_{i} t\left(z_{i}, u_{j}^{C}\right) t\left(z_{i}, u_{k}^{B}\right)\right) .
$$


ДокАЗАТЕЛьСтво. Воспользуемся результатом работы [20], где скалярное произведение твистованнного и обычного on-shell векторов Бете было получено в виде детерминанта матрицы размера $(a+b) \times(a+b)$. Его можно записать в блочной форме:

$$
S_{a, b}=\widetilde{H} \operatorname{det}_{a+b}\left(\begin{array}{ll}
A & B \\
C & D
\end{array}\right),
$$

где блок $A$ имеет размер $a \times a$, блок $B$ - размер $a \times b$, блок $C-$ размер $b \times a$ и блок $D-$ размер $b \times b$. Явные представления для блоков таковы:

$$
\begin{aligned}
A_{j k} & =h\left(u_{k}^{B}, \bar{u}^{B}\right)\left(t\left(u_{j}^{C}, u_{k}^{B}\right) \frac{f\left(\bar{v}^{B}, u_{k}^{B}\right) h\left(\bar{u}^{C}, u_{k}^{B}\right)}{f\left(\bar{v}^{C}, u_{k}^{B}\right) h\left(\bar{u}^{B}, u_{k}^{B}\right)}+\frac{\kappa_{2}}{\kappa_{1}} t\left(u_{k}^{B}, u_{j}^{C}\right) \frac{h\left(u_{k}^{B}, \bar{u}^{C}\right)}{h\left(u_{k}^{B}, \bar{u}^{B}\right)}\right), \\
B_{j k} & =\frac{\kappa_{2}}{\kappa_{1}} t\left(v_{k}^{C}, u_{j}^{C}\right) h\left(v_{k}^{C}, \bar{u}^{C}\right) \\
C_{j k} & =h\left(u_{k}^{B}, \bar{u}^{B}\right) \frac{g\left(u_{k}^{B}, \bar{v}^{B}\right)}{g\left(u_{k}^{B}, \bar{v}^{C}\right)}\left(g\left(u_{k}^{B}, v_{j}^{C}\right)+\frac{\kappa_{1}}{\kappa_{3} h\left(v_{j}^{C}, u_{k}^{B}\right)}\right), \\
D_{j k} & =\delta_{j k} h\left(v_{k}^{C}, \bar{u}^{B}\right) \frac{g\left(v_{k}^{C}, \bar{v}^{B}\right)}{g\left(v_{k}^{C}, \bar{v}_{k}^{C}\right)}\left(1-\frac{\kappa_{2}}{\kappa_{3}} \frac{f\left(v_{k}^{C}, \bar{u}^{C}\right)}{f\left(v_{k}^{C}, \bar{u}^{B}\right)}\right) .
\end{aligned}
$$

Множитель $\widetilde{H}$ задан выражением

$$
\widetilde{H}=\Delta_{a}^{\prime}\left(\bar{u}^{C}\right) \Delta_{a}\left(\bar{u}^{B}\right) \Delta_{b}^{\prime}\left(\bar{v}^{C}\right) \Delta_{b}\left(\bar{v}^{C}\right) g\left(\bar{v}^{C}, \bar{u}^{B}\right) .
$$

Воспользуемся хорошо известной формулой для детерминанта блочной матрицы,

$$
\operatorname{det}_{a+b}\left(\begin{array}{ll}
A & B \\
C & D
\end{array}\right)=\operatorname{det}_{b} D \underset{a}{\operatorname{det}}\left(A-B D^{-1} C\right) .
$$

Пусть $F=B D^{-1} C$. Заметим, что блок $D$ является диагональным: $D_{j k}=\delta_{j k} d_{k}$. Тогда получим

$$
F_{j k}=\sum_{\ell=1}^{b} \frac{1}{d_{\ell}} B_{j l} C_{l k} .
$$

Подставляя сюда все явные выражения, находим

$$
\begin{aligned}
& F_{j k}=\frac{\kappa_{2}}{\kappa_{1}} h\left(u_{k}^{B}, \bar{u}^{B}\right) \frac{g\left(u_{k}^{B}, \bar{v}^{B}\right)}{g\left(u_{k}^{B}, \bar{v}^{C}\right)} \times \\
& \times \sum_{\ell=1}^{b}\left(g\left(u_{k}^{B}, v_{\ell}^{C}\right)+\frac{\kappa_{1}}{\kappa_{3} h\left(v_{\ell}^{C}, u_{k}^{B}\right)}\right) t\left(v_{\ell}^{C}, u_{j}^{C}\right) \frac{h\left(v_{\ell}^{C}, \bar{u}^{C}\right)}{h\left(v_{\ell}^{C}, \bar{u}^{B}\right)} \frac{g\left(v_{\ell}^{C}, \bar{v}_{\ell}^{C}\right)}{g\left(v_{\ell}^{C}, \bar{v}^{B}\right)}\left(1-\frac{\kappa_{2}}{\kappa_{3}} \frac{f\left(v_{\ell}^{C}, \bar{u}^{C}\right)}{f\left(v_{\ell}^{C}, \bar{u}^{B}\right)}\right)^{-1} .
\end{aligned}
$$

Введем контурный интеграл вокруг бесконечно удаленной точки:

$$
\begin{aligned}
I_{j k} & =\frac{\kappa_{2}}{\kappa_{1}} h\left(u_{k}^{B}, \bar{u}^{B}\right) \frac{g\left(u_{k}^{B}, \bar{v}^{B}\right)}{g\left(u_{k}^{B}, \bar{v}^{C}\right)} \frac{1}{2 \pi i c} \times \\
& \times \oint_{|z| \rightarrow \infty}\left(g\left(u_{k}^{B}, z\right)+\frac{\kappa_{1}}{\kappa_{3} h\left(z, u_{k}^{B}\right)}\right) t\left(z, u_{j}^{C}\right) \frac{h\left(z, \bar{u}^{C}\right)}{h\left(z, \bar{u}^{B}\right)} \frac{g\left(z, \bar{v}^{C}\right)}{g\left(z, \bar{v}^{B}\right)}\left(1-\frac{\kappa_{2}}{\kappa_{3}} \frac{f\left(z, \bar{u}^{C}\right)}{f\left(z, \bar{u}^{B}\right)}\right)^{-1} .
\end{aligned}
$$

Очевидно, этот интеграл равен нулю. С другой стороны, он равен сумме вычетов внутри контура интегрирования. 
Имеются четыре типа таких вкладов:

- вклад полюсов $z=v_{\ell}^{C}, \ell=1, \ldots, b$, очевидно, что он совпадает с $F_{j k}$;

- вклад полюсов $z=u_{k}^{B}$, обозначим его через $P_{j k}^{(1)}$;

- вклад полюсов $z=u_{k}^{B}-c$, обозначим его через $P_{j k}^{(2)}$;

- вклад полюсов, расположенных в корнях $z_{i}, i=1, \ldots, 2 a$, уравнения (3.2), обозначим его через $P_{j k}^{(3)}$.

Отсюда имеем

$$
0=F_{j k}+P_{j k}^{(1)}+P_{j k}^{(2)}+P_{j k}^{(3)} .
$$

Явное вычисление показывает, что $P_{j k}^{(1)}+P_{j k}^{(2)}=-A_{j k}$. Подставляя это равенство в (3.12), мы приходим к формуле

$$
\operatorname{det}_{a+b}\left(\begin{array}{cc}
A & B \\
C & D
\end{array}\right)=\underset{b}{\operatorname{det}} D \underset{a}{\operatorname{det}} P_{j k}^{(3)},
$$

где

$$
P_{j k}^{(3)}=\frac{\kappa_{3}}{\kappa_{1}} h\left(u_{k}^{B}, \bar{u}^{B}\right) \frac{g\left(\bar{v}^{B}, u_{k}^{B}\right)}{g\left(\bar{v}^{C}, u_{k}^{B}\right)} \sum_{i=1}^{2 a} \alpha_{i} t\left(z_{i}, u_{j}^{C}\right)\left(g\left(u_{k}^{B}, z_{i}\right)+\frac{\kappa_{1}}{\kappa_{3} h\left(z_{i}, u_{k}^{B}\right)}\right),
$$

а $\alpha_{i}$ заданы равенством (3.3). Подставляя сюда $\operatorname{det}_{b} D$, мы немедленно воспроизводим представление (3.4).

\section{4. ЗАКЛЮЧЕНИЕ}

В представленной работе мы получили новое детерминантное представление для скалярного произведения твистованного и обычного on-shell векторов Бете в моделях с $\mathfrak{g l}(2 \mid 1)$-симметрией. Отметим, что данное представление содержит детерминант матрицы размера $a \times a$, в то время как представление, полученное в работе [20], содержит детерминант матрицы размера $(a+b) \times(a+b)$. Таким образом, новое представление является более удобным для численного анализа формфакторов и корреляционных функций.

Также заслуживает внимания новое представление (3.8) для скалярного произведения в моделях с $\mathfrak{g k}(2)$-симметрией. Как известно [30], коэффициенты асимптотического разложения корреляционных функций выражаются через скалярное произведение (3.8). Однако для этого следует вычислить термодинамический предел, в котором $\# \bar{u}^{C}=\# \bar{u}^{B} \rightarrow \infty$. Формула (3.8) открывает новые возможности в этом направлении.

\section{Список литературы}

[1] Е. К. Склянин, Л.А. Тахтаджян, Л.Д. Фаддеев, “Квантовый метод обратной задачи. I", ТМФ, 40:2 (1979), 194-220.

[2] V.E. Korepin, N. M. Bogoliubov, A. G. Izergin, Quantum Inverse Scattering Method and Correlation Functions, Cambridge Univ. Press, Cambridge, 1993.

[3] L. D. Faddeev, "How the algebraic Bethe ansatz works for integrable models", Symmétries quantiques [Quantum Symmetries], Proceedings of the Les Houches Summer School, Session LXIV (Les Houches, France, August 1 -September 8, 1995), eds. A. Connes, K. Gawedzki, J. Zinn-Justin, North-Holland, Amsterdam, 1998, 149-219. 
[4] V.E. Korepin, "Calculation of norms of Bethe wave functions", Commun. Math. Phys., 86:3 (1982), 391-418.

[5] A. G. Izergin, V. E. Korepin, "The quantum inverse scattering method approach to correlation functions", Commun. Math. Phys., 94:1 (1984), 67-92.

[6] А.Г. Изергин, "Статистическая сумма шестивершинной модели в конечном объеме", Докл. АН СССР, 297:2 (1987), 331-333.

[7] Н. А. Славнов, "Вычисление скалярных произведений волновых функций и формфакторов в рамках алгебраического анзаца Бете”, ТМФ, 79:2 (1989), 232-240.

[8] N. Kitanine, J. M. Maillet, V. Terras, "Correlation functions of the $X X Z$ Heisenberg spin-1/2 chain in a magnetic field", Nucl. Phys. B, 567:3 (2000), 554-582, arXiv: math-ph/9907019.

[9] N. Kitanine, J. M. Maillet, N. A. Slavnov, V. Terras, "Spin-spin correlation functions of the $X X Z-1 / 2$ Heisenberg chain in a magnetic field", Nucl. Phys. B, 641:3 (2002), 487-518, arXiv: hep-th/0201045.

[10] N. Kitanine, K. K. Kozlowski, J. M. Maillet, N. A. Slavnov, V. Terras, "Algebraic Bethe ansatz approach to the asymptotic behavior of correlation functions", J. Stat. Mech., 2009:4 (2009), P04003, 66 pp., arXiv: 0808.0227.

[11] F. Göhmann, A. Klümper, A. Seel, "Integral representations for correlation functions of the $X X Z$ chain at finite temperature", J. Phys. A: Math. Gen., 37:31 (2004), 7625-7652, arXiv: hep-th/0405089.

[12] F. Göhmann, A. Klümper, A. Seel, "Integral representation of the density matrix of the $X X Z$ chain at finite temperatures", J. Phys. A: Math. Gen., 38:9 (2005), 1833-1841, arXiv: cond-mat/0412062.

[13] A. Seel, T. Bhattacharyya, F. Göhmann, A. Klümper, "A note on the spin- $1 / 2 X X Z$ chain concerning its relation to the Bose gas", J. Stat. Mech., 2007:8 (2007), P08030, 10 pp., arXiv: 0705.3569.

[14] J.S. Caux, J.M. Maillet, "Computation of dynamical correlation functions of Heisenberg chains in a magnetic field", Phys. Rev. Lett., 95:7 (2005), 077201, 3 pp., arXiv: cond-mat/0502365.

[15] R. G. Pereira, J. Sirker, J. S. Caux, R. Hagemans, J. M. Maillet, S. R. White, I. Affleck, "Dynamical spin structure factor for the anisotropic spin-1/2 Heisenberg chain", Phys. Rev. Lett., 96:25 (2006), 257202, 4 pp., arXiv: cond-mat/0603681.

[16] R. G. Pereira, J. Sirker, J. S. Caux, R. Hagemans, J. M. Maillet, S. R. White, I. Affleck, "Dynamical structure factor at small $q$ for the $X X Z$ spin-1/2 chain", J. Stat. Mech., 2007:8 (2007), P08022, 64 pp., arXiv: 0706.4327.

[17] J. S. Caux, P. Calabrese, N. A. Slavnov, "One-particle dynamical correlations in the one-dimensional Bose gas", J. Stat. Mech., 2007:1 (2007), P01008, arXiv: cond-mat/0611321.

[18] S. Belliard, S. Pakuliak, E. Ragoucy, N. A. Slavnov, "The algebraic Bethe ansatz for scalar products in SU(3)-invariant integrable models", J. Stat. Mech., 2012:10 (2012), P10017, 25 pp., arXiv: 1207.0956.

[19] N. A. Slavnov, "Scalar products in $G L(3)$-based models with trigonometric $R$-matrix. Determinant representation", J. Stat. Mech., 2015:3 (2015), P03019, 25 pp., arXiv: 1501.06253.

[20] A. Hutsalyuk, A. Lyashik, S. Pakuliak, E. Ragoucy, N. A. Slavnov, "Scalar products of Bethe vectors in models with $\mathfrak{g l}(2 \mid 1)$ symmetry 2. Determinant representation", J. Phys. A, 50:3 (2017), 034004, 22 pp., arXiv: 1605.09189.

[21] B. Pozsgay, W.-V. van G. Oei, M. Kormos, "On form factors in nested Bethe Ansatz systems", J. Phys. A: Math. Gen., 2012:46, 465007, 34 pp., arXiv: 1204.4037.

[22] S. Belliard, S. Pakuliak, E. Ragoucy, N. A. Slavnov, "Form factors in SU(3)-invariant integrable models", J. Stat. Mech., 2013:4 (2013), P04033, 16 pp., arXiv: 1211.3968.

[23] S. Pakuliak, E. Ragoucy, N. A. Slavnov, "Form factors in quantum integrable models with GL(3)-invariant R-matrix", Nucl. Phys. B, 881 (2014), 343-368, arXiv: 1312.1488. 
[24] S. Pakuliak, E. Ragoucy, N. A. Slavnov, "Zero modes method and form factors in quantum integrable models", Nucl. Phys. B, 893 (2015), 459-481, arXiv: 1412.6037.

[25] S. Pakuliak, E. Ragoucy, N. A. Slavnov, "GL(3)-based quantum integrable composite models. II. Form factors of local operators", SIGMA, 11 (2015), 064, 18 pp., arXiv: 1502.01966.

[26] A. Hustalyuk, A. Liashyk, S. Z. Pakulyak, E. Ragoucy, N. A. Slavnov, "Form factors of the monodromy matrix entries in $\mathfrak{g l}(2 \mid 1)$-invariant integrable models", Nucl. Phys. B, 911 (2016), 902-927, arXiv: 1607.04978.

[27] J. Fuksa, N. A. Slavnov, "Form factors of local operators in supersymmetric quantum integrable models", J. Stat. Mech., 2017:4 (2017), 043106, 21 pp., arXiv: 1701.05866.

[28] П. П. Кулиш, Е. К. Склянин, "О решениях уравнения Янга-Бакстера”, Зап. научн. сем. ЛОМИ, 95 (1980), 129-160.

[29] S. Pakuliak, E. Ragoucy, N. A. Slavnov, "Bethe vectors for models based on super-Yangian $Y(\mathfrak{g l}(m \mid n))$ ", J. Integrab. Syst., 2:1 (2017), 1-31, arXiv: 1604.02311.

[30] A. Hutsalyuk, A. Lyashik, S. Pakuliak, E. Ragoucy, N. A. Slavnov, "Multiple actions of the monodromy matrix in $\mathfrak{g l}(2 \mid 1)$-invariant integrable models", SIGMA, 12 (2016), 099, 22 pp., arXiv: 1605.06419.

[31] N. Kitanine, K. K. Kozlowski, J. M. Maillet, N. A. Slavnov, V. Terras, "A form factor approach to the asymptotic behavior of correlation functions", J. Stat. Mech., 2011:12 (2011), P12010, 28 pp., arXiv: 1110.0803.

Поступила в редакцию 23.08.2018 